\title{
Stabilization of a class of fractional-order nonautonomous systems using quadratic Lyapunov functions
}

\author{
Quan $X u^{1,2,3 *}\left(\mathbb{D}\right.$, Shengxian Zhuang ${ }^{3}$, Xiaohui $X^{2,4}$, Chang $C^{3}$ and Yankun $X_{i a}^{5}$
}

\author{
"Correspondence: \\ quanxnjd@sina.com \\ 'School of Technology, Xihua \\ University, Chengdu, 610039, China \\ ${ }^{2}$ Key Laboratory of Fluid and Power \\ Machinery, Ministry of Education, \\ Xihua University, Chengdu, 610039, \\ China \\ Full list of author information is \\ available at the end of the article
}

\begin{abstract}
In this paper, we aim to solve the stabilization problem for a large class of fractional-order nonautonomous systems via linear state feedback control and adaptive control. By constructing quadratic Lyapunov functions and utilizing a new property for Caputo fractional derivative we derive some sufficient conditions for the global asymptotical stabilization of a class of fractional-order nonautonomous systems. We give two illustrative examples to validate the effectiveness of the theoretical results.
\end{abstract}

Keywords: fractional calculus; state feedback control; adaptive control; quadratic Lyapunov functions; fractional-order nonautonomous systems

\section{Introduction}

Fractional calculus, as a mathematical tool dealing with derivatives and integrals of arbitrary orders, has played a central role in physics [1], differential and integral equations [2], signal processing [3], human relationships [4], image encryption [5], thermal conductivity [6], electrical circuits [7], dynamical models [8], nonlinear control systems [9], complex networks [10], and so on. One of very important areas of application is nonlinear control systems. In particular, stability analysis and stabilization are of theoretical and practical importance for control systems, certainly including fractional-order systems. The early studies on the stability of fractional-order systems mainly concentrated on the linear cases, and many well-known results have been obtained. However, the stability analysis of fractional-order nonlinear systems remains an open problem. More details on the development of stability of fractional-order systems can be found in [11-15].

Recently, the stability and stabilization problem of a class of fractional-order nonlinear systems has attracted increasing interest of scholars [16-20]. In these literatures, it has been shown that most of the well-known chaotic systems can be modeled as this kind of fractional-order nonlinear systems (so-called semilinear systems), where the nonlinear vector field can be separated into linear and nonlinear parts. Moreover, the Mittag-Leffler function, the Laplace transform, and the Gronwall lemma are the main techniques used to prove the stability. It was shown that these techniques are neither simple nor straightforward and the proofs of the corresponding theorems are very complex. It should be also noted that the stability results in [16-19] are local, and all of the stability results in [16-20] 
are valid only for fractional-order autonomous systems. However, as we know, the stability results for fractional-order nonautonomous systems, including chaotic and nonchaotic systems, are still relatively few.

In integer-order nonautonomous systems, the Lyapunov direct method is an effective way to analyze the stability of a system. Motivated by the application of fractional calculus in nonlinear systems, the Lyapunov direct method has been extended to fractionalorder systems by Li et al. [21, 22]. Similarly, Baleanu et al. [23] and Wu et al. [24] extended the theorem to fractional-order functional systems and fractional-order discrete systems. The fractional Lyapunov method generalizes the idea that the stability condition is derived by constructing a suitable Lyapunov function and calculating its fractional derivative. However, it is not an easy task to apply this method in the stability analysis of a general fractional-order nonlinear system. In [25], it has been demonstrated that fractional derivatives of noninteger orders cannot satisfy the Leibniz rule. So far, there are no techniques available to calculate the fractional derivative of a general composite Lyapunov function.

Recently, some efforts have been devoted to application of the fractional Lyapunov direct method in stability analysis of fractional-order systems. Especially, in [26], a simple Lyapunov function $V=\lambda^{T} x, \lambda>0$ has been proposed to solve the stabilization problem for fractional-order linear positive systems. By constructing some suitable stochastic Lyapunov functions Agarwal et al. [27] established some sufficient conditions for two types of stability of stochastic differential equations. In [28], based on the frequency distributed fractional integrator model, a fractional-order system is transformed into an equivalent integer-order system, and then similar stability results as those for integer-order systems are obtained by using quadratic Lyapunov functions. Hu et al. [29] considered an integerorder derivative instead of the fractional-order derivative of a Lyapunov function to prove the revised Lyapunov stability theorems. Nevertheless, the proposed Lyapunov functions [21-24, 26-29] are valid only for some fractional-order systems with special characteristics.

In classic Lyapunov theory, the quadratic form is one of the most commonly used Lyapunov functions for general integer-order nonlinear systems. Motivated by this, Aguila-Camacho et al. [30] and Duarte-Mermoud et al. [31] introduced two new inequalities for estimating the Caputo fractional derivative of a quadratic function and a common quadratic function, respectively. Thus, wecan try to prove the stability for general fractional-order nonlinear systems by using common quadratic Lyapunov functions.

Quite recently, Liu et al. [32] studied the stability of a class of fractional nonlinear systems using the fractional Lyapunov direct method and a new lemma proposed in [30, 31]. In this paper, we aim to solve the stabilization problem for such fractional-order systems via linear state feedback control and adaptive control.

The main contributions of this paper are as follows. First, constructing quadratic Lyapunov functions and using a new property for the Caputo derivative, we respectively investigate the stabilization of a class of fractional-order nonautonomous systems via state feedback control and adaptive control. Then, we derive some sufficient conditions for the global asymptotical stabilization. Note that using the existing results in [16-20], we may draw a wrong conclusion about asymptotic stability for our nonautonomous model. Moreover, asymptotic stability analysis of fractional-order nonautonomous systems is more difficult. This technical difficulty can be overcome by the fractional Lyapunov direct method $[21,22]$ and a new property for the Caputo derivative. Further, we also prove that quadratic 
Lyapunov functions are always valid for our model. Differing from [16-20], our results can only be applied not only to fractional-order autonomous systems, but also to fractionalorder nonautonomous systems. In practice, we can easily realize the stabilization of this kind of fractional-order systems based on our criteria.

The remainder of this paper is organized as follows. In Section 2, we introduce some useful definitions, properties, and preliminaries for fractional calculus and fractional-order systems. In Section 3, we give three stabilization criteria for a class of fractional-order nonautonomous systems. In Section 4, we provide three numerical examples to illustrate the effectiveness of the theoretical results. Finally, we give some conclusions and further work in Section 5.

Notions Let $\mathbb{R}=(-\infty,+\infty), \mathbb{R}^{n}$ be the $n$-dimensional Euclidean space, and let $\mathbb{R}^{m \times n}$ be the space of $m \times n$ real matrices; $\|x\|=\sqrt{x^{T} x}$ is the two-norm of a vector $x,\|P\|=$ $\sqrt{\lambda_{\max }\left(P^{T} P\right)}$ is the two-norm of a matrix $P$, and $\lambda_{\min }(\cdot)\left(\lambda_{\max }(\cdot)\right)$ denotes the minimum (maximum) eigenvalue of the corresponding matrix.

\section{Preliminaries}

In this paper, we consider the Caputo definition of fractional derivative, which is most popular in engineering applications.

Definition 2.1 ([33]) The uniform formula of a fractional integral with $0<\alpha<1$ is given by

$$
I_{t}^{\alpha} f(t)=\frac{1}{\Gamma(\alpha)} \int_{t_{0}}^{t}(t-\tau)^{\alpha-1} f(\tau) d \tau
$$

where $t \geq t_{0}, f(t)$ is an arbitrary integrable function, $I_{t}^{\alpha}$ is the fractional integral operator, $\Gamma(\alpha)=\int_{0}^{\infty} t^{\alpha-1} \exp (-t) d t$ is the gamma function, and $\exp (\cdot)$ is the exponential function.

Definition 2.2 ([33]) The Caputo fractional derivative with fractional order $\alpha$ can be expressed as

$$
{ }_{t_{0}}^{C} D_{t}^{\alpha} f(t)=\frac{1}{\Gamma(n-\alpha)} \int_{t_{0}}^{t} \frac{f^{n}(\tau)}{(t-\tau)^{\alpha-n+1}} d \tau,
$$

where $[\alpha]$ denotes the integer part of $\alpha$. Throughout this paper, we choose the notion $D_{t}^{\alpha}$ as the Caputo fractional derivative operator ${ }_{t_{0}}^{C} D_{t}^{\alpha}$. In the following, unless otherwise stated, we consider $\alpha \in(0,1)$.

The Laplace transform of the Caputo fractional derivative of a function can be written as

$$
\mathcal{L}\left\{D_{t}^{\alpha} f(t)\right\}=s^{\alpha} F(s)-s^{\alpha-1} f\left(t_{0}\right)
$$

where $\alpha \in(0,1), s$ is the variable in the Laplace domain, $\mathcal{L}\{\cdot\}$ denotes the Laplace transform, $f\left(t_{0}\right)$ is the initial value, and $F(s)$ is the Laplace transform of $f(t)$.

Property $2.1([33]) I_{t}^{\alpha} D_{t}^{\alpha} f(t)=f(t)-f\left(t_{0}\right), \forall t \geq t_{0}$. 
According to the definition of the Caputo derivative, a general fractional-order nonlinear and nonautonomous system can be characterized by

$$
D_{t}^{\alpha} x(t)=f(t, x)
$$

where $\alpha \in(0,1)$, and $t$ denotes the time.

For the stability analysis of system (2.4), the fractional Lyapunov direct method has been proposed [21, 22], which is stated in Lemma 2.1.

Remark 2.1 From [22] we know that system (2.4) has the same equilibrium points as the integer-order system $\dot{x}(t)=f(t, x)$.

Definition 2.3 A continuous function $\gamma:[0, t] \rightarrow[0,+\infty]$ is said to belong to class $\mathcal{K}$ if it is strictly increasing and $\gamma(0)=0$.

Lemma 2.1 ([21,22]) Let $x=0$ be an equilibrium point for the fractional-order nonlinear system (2.4). Let $V(t, x)$ be a Lyapunov function, and let $\gamma_{i}(i=1,2,3)$ be functions of class $\mathcal{K}$ such that
(a) $\quad \gamma_{1}(\|x\|) \leq V(t, x) \leq \gamma_{2}(\|x\|)$,
(b) $D_{t}^{\beta} V(t, x) \leq-\gamma_{3}(\|x\|)$,

where $\beta \in(0,1)$. Then, the equilibrium point $x=0$ is asymptotically stable.

Remark 2.2 For a quadratic positive definite function $V=x^{T} P x$, the following inequalities are always true:

$$
\lambda_{\min }(P)\|x\|^{2} \leq x^{T} P x \leq \lambda_{\max }(P)\|x\|^{2} .
$$

Obviously, condition (a) of Lemma 2.1 is naturally satisfied if we choose $V=x^{T} P x$ as a Lyapunov function.

According to [31], calculating the fractional derivative of the product of two functions implies evaluating an infinite sum. Obviously, this is not an easy task. This is also the main reason that fractional theory, especially fractional Lyapunov direct theory, is not as popular as it should be. Recently, a new property for the Caputo derivative is stated in Lemma 2.2 [31], which can facilitate estimating the fractional derivative of a common quadratic Lyapunov function.

Lemma 2.2 ([31]) Let $x(t) \in \mathbb{R}^{n}$ be a vector of differentiable functions. Then, the following inequality holds:

$$
D_{t}^{\alpha} x^{T}(t) P x(t) \leq 2 x^{T}(t) P D_{t}^{\alpha} x(t) \quad \forall \alpha \in(0,1], \forall t \geq t_{0},
$$

where $P \in \mathbb{R}^{n \times n}$ is a constant symmetric positive definite matrix. 
Definition 2.4 ([22]) The Mittag-Leffler functions in one parameter and two parameters are defined as

$$
\begin{aligned}
& E_{\alpha}(z)=\sum_{n=0}^{\infty} \frac{z^{n}}{\Gamma(n \alpha+1)}, \\
& E_{\alpha, \beta}(z)=\sum_{n=0}^{\infty} \frac{z^{n}}{\Gamma(n \alpha+\beta)},
\end{aligned}
$$

where $z \in \mathbb{C}, \alpha>0, \beta>0, E_{\alpha, 1}(z)=E_{\alpha}(z)$, and $E_{1,1}(z)=\mathrm{e}^{z}$.

The Laplace transform for the Mittag-Leffler function with two parameters is written as follows:

$$
\mathcal{L}\left\{t^{\beta-1} E_{\alpha, \beta}\left(-k t^{\alpha}\right)\right\}=\frac{s^{\alpha-\beta}}{s^{\alpha}+k}, \quad \mathcal{R}(s)>|k|^{\frac{1}{\alpha}},
$$

where $t \geq 0, \mathcal{R}(s)$ is the real part of $s$, and $k \in \mathbb{R}$.

\section{Main results}

In this section, by constructing quadratic Lyapunov functions we derive some stabilization criteria for a class of fractional-order nonautonomous systems based on the fractional Lyapunov direct method and other inequality techniques.

Consider the following fractional-order nonautonomous system:

$$
D_{t}^{\alpha} x(t)=f(t, x)=A x+g(t, x)
$$

where $\alpha \in(0,1), x=\left(x_{1}, x_{2}, \ldots, x_{n}\right)^{T} \in \mathbb{R}^{n}$ represents the state vector of the system, $A \in$ $\mathbb{R}^{n \times n}$ is a constant matrix, and $g:\left[t_{0},+\infty\right] \times \mathbb{R}^{n} \rightarrow \mathbb{R}^{n}$ is a nonlinear vector function.

Remark 3.1 In general, a differential equation is called semilinear if it consists of the sum of a well-understood linear part plus a lower-order nonlinear part. It is well known that many real systems in engineering and science can be modeled as semilinear systems [34]. Therefore, our model can describe a large class of fractional-order physical systems, including linear systems with nonlinear perturbation, chaotic systems, and so on. Our major aim is to show how a simple state feedback controller can be designed to stabilize this type of systems if the nonlinear part satisfies a constraint.

Assumption 3.1 For a nonlinear function $g, g(t, 0)=0$ and there exists a constant $\varepsilon>0$ such that $\|g(t, x)\| \leq \varepsilon\|x\|$ for $x \in \mathbb{R}^{n}$ and $t \in\left[t_{0},+\infty\right]$.

Remark 3.2 In fact, Assumption 3.1 is the so-called global Lipschitz condition. It seems that this assumption is not easy to satisfy in general nonlinear systems. However, as stated in Remark 3.1, our model has potential applications in robust stabilization of linear systems with nonlinear perturbation and chaotic control and synchronization. According to Remark 3 in [35], if a system is Lipschitz, then it can be made contracting by means of a simple (static) state feedback, implying the possibility of designing simple observers/controllers. Fortunately, since the chaotic is bounded, we can easily verify that 
many well-known chaotic systems, such as Chua's circuit, Arneodo's system, Lorenz's system, Duffing's oscillator, Chen's system, etc., all satisfy this assumption.

Quite recently, in [32], conditions for the asymptotical stability of system (3.1) have been derived by using the fractional Lyapunov direct method and Lemma 2.2.

Lemma 3.1 ([32]) Suppose that Assumption 3.1 holds. System (3.1) is globally asymptotically stable about its equilibrium point if there exists a symmetric matrix $P>0, P \in \mathbb{R}^{n \times n}$, such that

$$
\left(\begin{array}{cc}
P A+A^{T} P+r \varepsilon^{2} I & P \\
P & -r I
\end{array}\right)<0,
$$

where $\varepsilon>0, r>0$, and I denotes identity matrix.

In this paper, we further study the stabilization of system (3.1) via linear state feedback control and adaptive control.

A controlled system with state feedback is characterized by

$$
D_{t}^{\alpha} x(t)=A x+g(t, x)+u=(A-K) x+g(t, x)=\tilde{A} x+g(t, x),
$$

where $\alpha \in(0,1), K \in \mathbb{R}^{n \times n}$ is a feedback gain matrix, and $\tilde{A}=A-K$. Thus, our aim is to select a suitable matrix $K$ such that the controlled system (3.3) is asymptotically stable.

Theorem 3.1 Suppose that Assumption 3.1 holds. The controlled system (3.3) with the feedback gain $K=Z Y^{-1}$ is globally asymptotically stable if there are suitable matrices $Y$ and $Z$ such that the following LMI condition holds:

$$
\left(\begin{array}{cc}
A Y+Y A^{T}-Z-Z^{T}-2 \varepsilon Y & I+\varepsilon r Y \\
I+\varepsilon r Y & -r I
\end{array}\right)<0,
$$

where $Y=P^{-1}>0, \varepsilon>0, r>0$, and I is the identity matrix.

Proof Substituting $\tilde{A}=A-B K$ for $A$ in (3.2), we easily obtain

$$
\left(\begin{array}{cc}
P A+A^{T} P-P K-K^{T} P+r \varepsilon^{2} I & P \\
P & -r I
\end{array}\right)<0 .
$$

By using Schur complements, (3.5) can be rewritten as

$$
\left(\begin{array}{cc}
P A+A^{T} P-P K-K^{T} P-2 \varepsilon P & P+\varepsilon r I \\
P+\varepsilon r I & -r I
\end{array}\right)<0 .
$$

Let $Y=P^{-1}$ and $Z=K P^{-1}$. Multiplying (3.6) by $\left(\begin{array}{ll}Y & 0 \\ 0 & I\end{array}\right)$ from the both sides, we can further transform the condition (3.6) into the LMI form (3.4). The proof is completed. 
Remark 3.3 It should be noted that the feasible set $Y$ and $Z$ satisfying (3.4) can be solved by using the LMI Toolbox in Matlab software. Thus, a feedback gain matrix $K$ can be further obtained by $K=Z Y^{-1}$. However, without any constraint on $K$, the feedback controller to stabilize a huge dimension system may be relatively complex and difficult to apply in practice.

Theorem 3.2 Suppose that Assumption 3.1 holds. If the feedback gain matrix $K$ is selected such that all eigenvalues of $\tilde{A}$ satisfy $\operatorname{Re} \lambda(\tilde{A})<0$ and the solution $P$ of the equation $P \tilde{A}+\tilde{A}^{T} P=-I$ satisfies $1-2 \varepsilon \lambda_{\max }(P)>0$, then the controlled system (3.3) is globally asymptotically stable about its equilibrium point, where I denotes the identity matrix.

Proof Consider the Lyapunov function candidate

$$
V(x)=x^{T} P x, \quad P>0, P \in \mathbb{R}^{n \times n} .
$$

Applying Lemma 2.2, the fractional derivative of Lyapunov function (3.7) along the trajectories of system (3.3) gives

$$
D_{t}^{\alpha} V \leq 2 x^{T} P D_{t}^{\alpha} x=x^{T}\left[P \tilde{A}+(P \tilde{A})^{T}\right] x+2 x^{T} P g(t, x) .
$$

From trivial algebra, using the Cauchy-Schwarz inequality, we have

$$
x^{T} P g(t, x) \leq\|x\|\|P\|\|g(t, x)\| \leq\|x\| \lambda_{\max }(P)\|g(t, x)\| .
$$

From Assumption 3.1 it follows that

$$
x^{T} P g(t, x) \leq\|x\| \lambda_{\max }(P) \varepsilon\|x\|=\varepsilon \lambda_{\max }(P) x^{T} x .
$$

Therefore, combing (3.8) and (3.10), we have

$$
D_{t}^{\alpha} V \leq x^{T}\left[P \tilde{A}+(P \tilde{A})^{T}\right] x+2 \varepsilon \lambda_{\max }(P) x^{T} x .
$$

Since all eigenvalues of $\tilde{A}$ satisfy $\operatorname{Re} \lambda(\tilde{A})<0$, there exists a unique positive definite solution $P$ of the equation $P \tilde{A}+\tilde{A}^{T} P=-I$. Next, from (3.11) we obtain

$$
D_{t}^{\alpha} V \leq-x^{T} x+2 \varepsilon \lambda_{\max }(P) x^{T} x=-\left(1-2 \varepsilon \lambda_{\max }(P)\right)\|x\|^{2} .
$$

Therefore, if $1-2 \varepsilon \lambda_{\max }(P)>0$ and the $\mathcal{K}$ functions $\gamma_{1}(\|x\|)=\lambda_{\min }(P)\|x\|^{2}$ and $\gamma_{2}(\|x\|)=$ $\lambda_{\max }(P)\|x\|^{2}, \gamma_{3}(\|x\|)=\left(1-2 \varepsilon \lambda_{\max }(P)\right)\|x\|^{2}$ are used, then all the conditions of Lemma 2.1 are satisfied. As a result, the equilibrium point of the system (3.3) is globally asymptotically stable. The proof is completed.

Next, the fractional adaptive law is designed to tune the feedback gain matrix $K$ of system (3.3). For simplification and without loss of generality, we assume that $K=$ $\operatorname{diag}\left(k_{1}(t), k_{2}(t), \ldots, k_{n}(t)\right)$. 
Theorem 3.3 Suppose that Assumption 3.1 holds. Then, the controlled system (3.3) is globally asymptotically stable about its equilibrium point under the following adaptive updating law:

$$
D_{t}^{\alpha} k_{i}(t)=\mu_{i} x_{i}^{2}, \quad i=1,2, \ldots, n,
$$

where $\mu_{i}$ are positive constants.

Proof Consider a Lyapunov function for system (3.3) as follows:

$$
V(t)=\frac{1}{2} x^{T} x+\sum_{i=1}^{n} \frac{1}{2 \mu_{i}}\left(k_{i}(t)-k_{i}^{*}\right)^{2}
$$

where $k_{i}^{*}(i=1,2, \ldots, n)$ are positive constants to be determined later.

Applying Lemma 2.2, the fractional derivative of Lyapunov function (3.14) along the trajectories of system (3.3) and the adaptive law (3.13) gives

$$
\begin{aligned}
D_{t}^{\alpha} V & \leq x^{T} D_{t}^{\alpha} x+\sum_{i=1}^{n} \frac{1}{\mu_{i}}\left(k_{i}-k_{i}^{*}\right) D_{t}^{\alpha} k_{i} \\
& =x^{T}[A x+g(t, x)-K x]+\sum_{i=1}^{n}\left(k_{i}-k_{i}^{*}\right) x_{i}^{2} \\
& =x^{T}[A x+g(t, x)-K x]+K x^{T} x-K^{*} x^{T} x \\
& =x^{T} A x+x^{T} g(t, x)-K^{*} x^{T} x \\
& =x^{T} \frac{A^{T}+A}{2} x+x^{T} g(t, x)-K^{*} x^{T} x,
\end{aligned}
$$

where $K^{*}=\operatorname{diag}\left(k_{1}^{*}, k_{2}^{*}, \ldots, k_{n}^{*}\right)$.

By the Cauchy-Schwarz inequality from Assumption 3.1 it follows that

$$
x^{T} g(t, x) \leq\|x\|\|g(t, x)\| \leq \varepsilon x^{T} x
$$

Combing (3.15) and (3.16), we obtain

$$
D_{t}^{\alpha} V \leq x^{T}\left(\frac{A^{T}+A}{2}+\varepsilon I-K^{*}\right) x .
$$

Choosing $k_{i}^{*}$ sufficiently large such that

$$
\lambda_{\max }\left(\frac{A^{T}+A}{2}\right)+\varepsilon-\min \left\{k_{i}^{*}\right\}+1 \leq 0,
$$

where $\min \left\{k_{i}^{*}\right\}$ denotes the minimum value of $k_{1}^{*}, k_{2}^{*}, \ldots, k_{n}^{*}$, we obtain

$$
D_{t}^{\alpha} V \leq-x^{T} x
$$

Obviously, there exists $p(t) \geq 0$ satisfying

$$
D_{t}^{\alpha} V(t)+p(t)=-x(t)^{T} x(t)
$$


Calculating the Laplace transform of (3.20), we obtain

$$
s^{\alpha} V(s)-s^{\alpha-1} V(0)+P(s)=-X(s)
$$

where $V(0) \geq 0$ is the initial value of $V(t)$, and $V(s), P(s)$, and $X(s)$ denote the Laplace transforms of $V(t), p(t)$, and $x(t)^{T} x(t)$, respectively.

Since $V(t) \geq \frac{1}{2} x(t)^{T} x(t)$, there exists a function $q(t) \geq 0$ such that

$$
V(t)=\frac{1}{2} x(t)^{T} x(t)+q(t)
$$

Calculating the Laplace transform of (3.22), we get

$$
V(s)=\frac{1}{2} X(s)+Q(s)
$$

where $Q(s)$ is the Laplace transform of $q(t)$.

Substituting (3.21) into (3.23), we have

$$
\begin{aligned}
X(s) & =\frac{2 s^{\alpha-1}}{s^{\alpha}+2} V(0)-\frac{2 s^{\alpha}}{s^{\alpha}+2} Q(s)-\frac{2}{s^{\alpha}+2} P(s) \\
& =\frac{2 s^{\alpha-1}}{s^{\alpha}+2} V(0)-2\left[\left(s \frac{s^{\alpha-1}}{s^{\alpha}+2}-1\right) Q(s)+Q(s)\right]-\frac{2}{s^{\alpha}+2} P(s) \\
& =\frac{2 s^{\alpha-1}}{s^{\alpha}+2} V(0)+\frac{4}{s^{\alpha}+2} Q(s)-2 Q(s)-\frac{2}{s^{\alpha}+2} P(s) .
\end{aligned}
$$

Calculating the inverse Laplace transform of (3.24), we obtain

$$
\begin{aligned}
x(t)^{T} x(t)= & 2 V(0) E_{\alpha}\left(-2 t^{\alpha}\right)-2 q(t)+4 q(t) * t^{\alpha-1} E_{\alpha, \alpha}\left(-2 t^{\alpha}\right) \\
& -2 p(t) * t^{\alpha-1} E_{\alpha, \alpha}\left(-2 t^{\alpha}\right)
\end{aligned}
$$

where $*$ is the convolution operator.

Since $t^{\alpha-1}, p(t), q(t)$, and $E_{\alpha, \alpha}\left(-2 t^{\alpha}\right)$ are nonnegative, we can derive from (3.25) that

$$
x(t)^{T} x(t) \leq 2 V(0) E_{\alpha}\left(-2 t^{\alpha}\right)+4 q(t) * t^{\alpha-1} E_{\alpha, \alpha}\left(-2 t^{\alpha}\right)
$$

By Definition 2.1 and Property 2.1, from (3.19) this yields

$$
I_{t}^{\alpha} D_{t}^{\alpha} V(t)=V(t)-V(0)=\frac{1}{\Gamma(\alpha)} \int_{0}^{t}(t-\tau)^{\alpha-1} D_{t}^{\alpha} V(\tau) d \tau \leq 0
$$

that is, $V(t) \leq V(0), t \geq 0$.

Combining the aforementioned situation, we arrive at

$$
0 \leq q(t) \leq V(t) \leq V(0)
$$

Thus, $q(t)$ is bounded.

Recall now that, for $0<\alpha \leq \beta<1$ and $\theta>0, E_{\alpha}\left(-\theta t^{\alpha}\right)$ and $t^{\beta-1} E_{\alpha, \beta}\left(-\theta t^{\alpha}\right)$ are both completely monotonic (see [22]). As a result, from (3.26) we obtain $\lim _{t \rightarrow+\infty} x(t)^{T} x(t)=0$, 
namely $\lim _{t \rightarrow+\infty} x(t)=0$. Considering (3.13), this implies that $\lim _{t \rightarrow+\infty} D_{t}^{\alpha} k_{i}=0$. Noting now that the Caputo fractional derivative of a constant is always zero, we immediately conclude that $k_{i}(i=1,2, \ldots, n)$ converges to a finite constant. Consequently, system (3.3) is stabilized by the adaptive law (3.13). The proof is completed.

Remark 3.4 It should be noted that the Lyapunov (3.14) is the function of two variables $x(t)$ and $k_{i}(t)$, and $D_{t}^{\alpha} V \leq-x(t)^{T} x(t)$. To prove the convergence of $x(t)$, condition (2.5) of Lemma 2.1 is not satisfied. In other words, the fractional Lyapunov direct method is not sufficient for proving the asymptotical stability of system (3.3) with adaptive law (3.13). It is well known that LaSalle's invariance principle, Barbalat's lemma, and other mathematical techniques can be used to solve the adaptive stability problem of integer-order nonlinear systems. However, these tools cannot be directly used in the fractional-order case. In this paper, the adaptive stability problem of fractional-order systems has been settled by utilizing the fractional Lyapunov function method combined with fractional inequality techniques, the Mittag-Leffler function, and the Laplace transform.

\section{Illustrative examples}

In this section, we give three illustrative examples to validate the theoretical results and use the predictor-corrector method [36] for numerical simulations.

Example 1 The fractional-order nonlinear nonautonomous parametrically excited Duffing oscillators is described by

$$
\begin{aligned}
& D_{t}^{\alpha} x_{1}=x_{2}, \\
& D_{t}^{\alpha} x_{2}=(1+\mu \sin (\omega t)) x_{1}-\gamma x_{2}-x_{1}^{3} .
\end{aligned}
$$

When the parameters are chosen as $\mu=0.5, \omega=1, \gamma=0.2$ and $\alpha=0.975$, system (4.1) has a chaotic attractor shown in Figure 1.

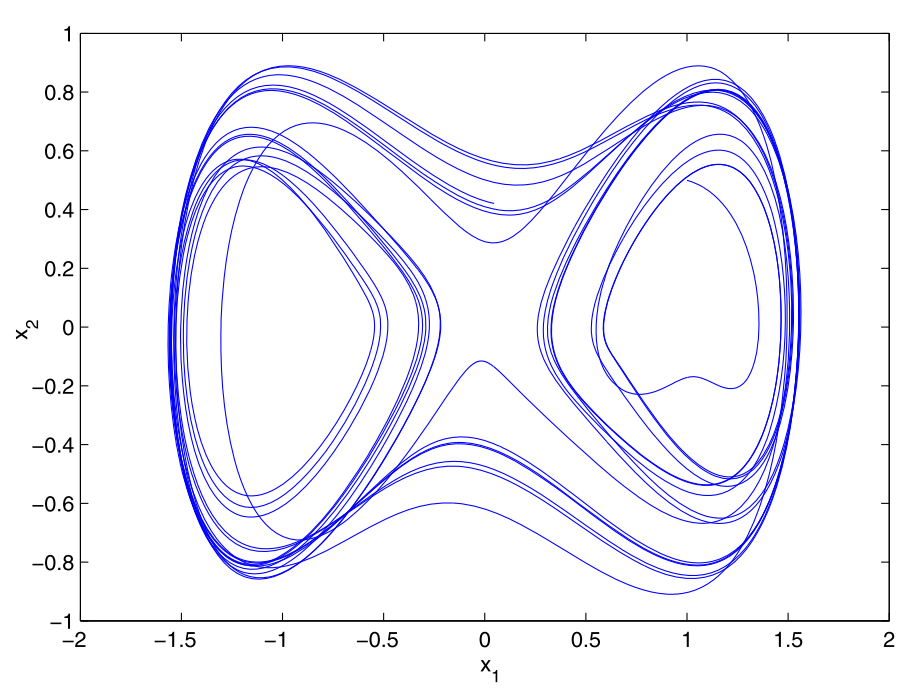

Figure 1 Chaotic attractor of system (4.1) with $\mu=0.5, \omega=1, \gamma=0.2$, and $\alpha=0.975$. 
System (4.1) can be rewritten as system (3.3), where

$$
A=\left(\begin{array}{cc}
0 & 1 \\
1 & -0.2
\end{array}\right), \quad g(t, x)=\left(\begin{array}{c}
0 \\
0.5 \sin (t) x_{1}-x_{1}^{3}
\end{array}\right)
$$

Then, we easily obtain

$$
\begin{aligned}
\|g(t, x)\| & =\sqrt{\left(0.5 \sin (t)-x_{1}^{2}\right)^{2} x_{1}^{2}} \\
& \leq\left|0.5 \sin (t)-x_{1}^{2}\right| \sqrt{x_{1}^{2}+x_{2}^{2}} \leq\left(0.5+x_{1}^{2}\right)\|x\| .
\end{aligned}
$$

Since the chaotic system is bounded, we easily get that $\left|x_{1}\right|<2$. Therefore, Assumption 3.1 is satisfied, and $\varepsilon=4.5$. Choosing $r=1$ and using the LMI Toolbox in MATLAB, it follows from condition (3.4) that

$$
\begin{aligned}
Y & =\left(\begin{array}{cc}
0.1233 & 0 \\
0 & 0.1233
\end{array}\right)>0, \quad Z=\left(\begin{array}{cc}
-0.2900 & 1.2887 \\
-1.5353 & -0.2653
\end{array}\right) \\
K & =\left(\begin{array}{cc}
-2.3515 & 10.4492 \\
-12.4492 & -2.1515
\end{array}\right) .
\end{aligned}
$$

Therefore, all the conditions of Theorem 3.1 are satisfied, and we can conclude that the controlled system is asymptotically stable. The simulation results shown in Figure 2 verify the theoretical analysis.

In addition, according to Theorem 3.2, we choose the feedback gain $K=\operatorname{diag}(-6,-5)$. By a simple calculation the eigenvalues of $\tilde{A}$, the unique solution $P$ of $P \tilde{A}+\tilde{A}^{T} P=-I$, and

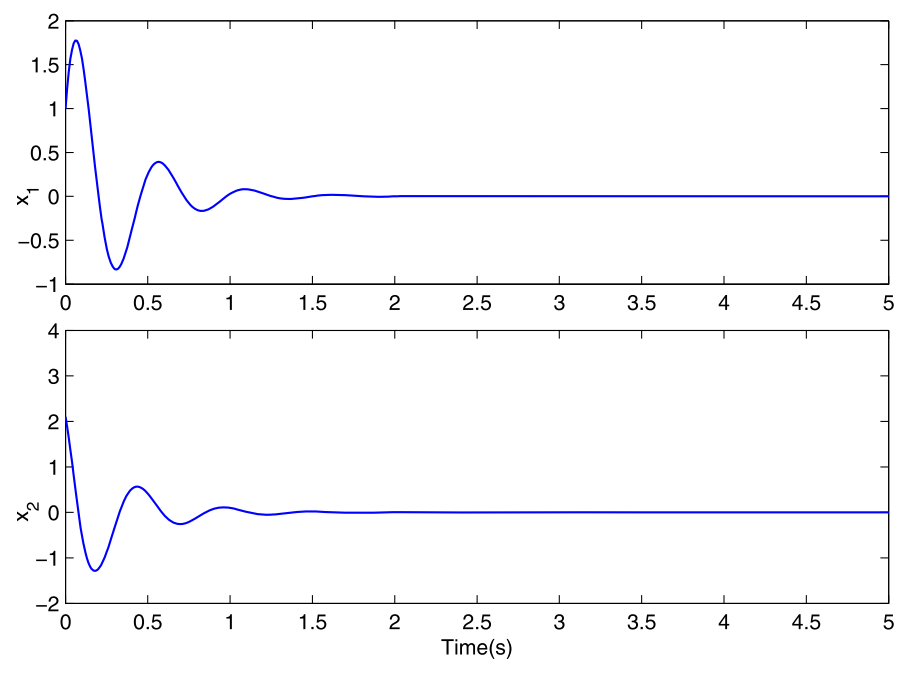

Figure 2 Asymptotic stabilization of system (4.1) with feedback gain (4.4). 


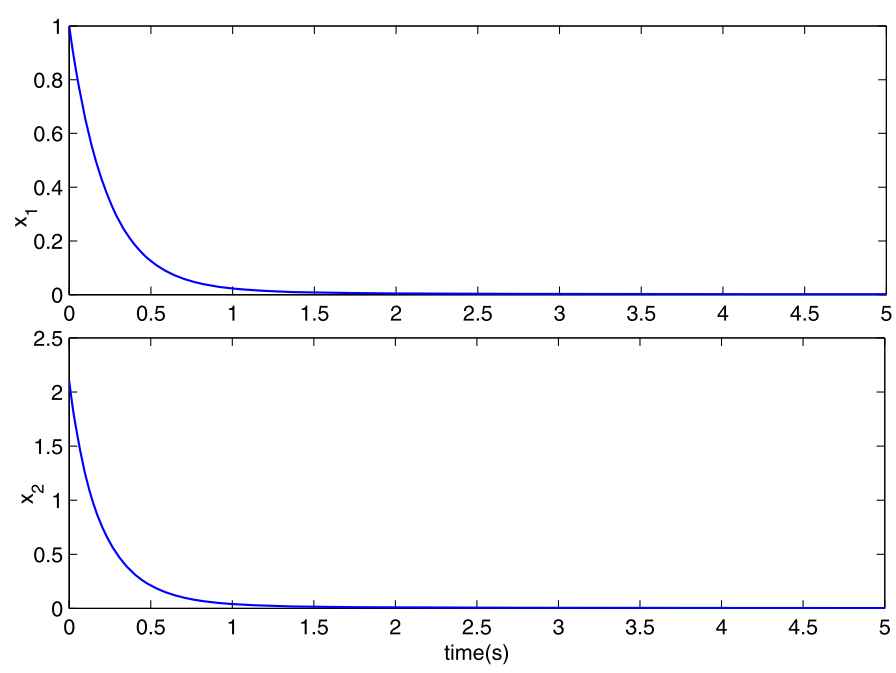

Figure 3 Asymptotic stabilization of system (4.1) with feedback gain $K=\operatorname{diag}(-6,-5)$.

the maximum eigenvalue of $P$ are given by

$$
\begin{aligned}
& \lambda_{1}=-6.677<0, \quad \lambda_{2}=-4.523<0, \\
& P=\left(\begin{array}{ll}
0.0861 & 0.0166 \\
0.0166 & 0.0993
\end{array}\right), \quad \lambda_{\max }(P)=0.1105, \\
& 1-2 \varepsilon \lambda_{\max }(P)=0.0055>0 .
\end{aligned}
$$

Therefore, all the conditions of Theorem 3.2 are satisfied, and we can conclude that the controlled system is asymptotically stable. The simulation results shown in Figure 3 verify the theoretical analysis.

Example 2 The fractional-order Arneodo system [37] is described by

$$
\begin{aligned}
& D_{t}^{\alpha} x_{1}=x_{2}, \\
& D_{t}^{\alpha} x_{2}=x_{3}, \\
& D_{t}^{\alpha} x_{3}=\beta_{1} x_{1}-\beta_{2} x_{2}-\beta_{3} x_{3}+\beta_{4} x_{1}^{3} .
\end{aligned}
$$

When the parameters are chosen as $\beta_{1}=5.5, \beta_{2}=3.5, \beta_{3}=0.8, \beta_{4}=-1$, and $\alpha=0.97$, system (4.6) displays the chaotic behavior shown in Figure 4. System (4.6) can be rewritten as system (3.3), where

$$
\begin{aligned}
& A=\left(\begin{array}{ccc}
0 & 1 & 0 \\
0 & 0 & 1 \\
5.5 & -3.5 & -0.8
\end{array}\right), \quad g(t, x)=\left(\begin{array}{c}
0 \\
0 \\
-x_{1}^{3}
\end{array}\right), \\
& \|g(t, x)\|=\sqrt{x_{1}^{6}} \leq x_{1}^{2}\|x\| .
\end{aligned}
$$

Since the chaotic system is bounded, Assumption 3.1 is satisfied. To suppress the chaos, the adaptive law (3.13) is designed to tune the feedback gains $k_{i}$. According to Theorem 3.3, we arbitrarily take $\left(\mu_{1}, \mu_{2}, \mu_{3}\right)=(3,4,1)$. The initial conditions of the state vector $x$ and the 

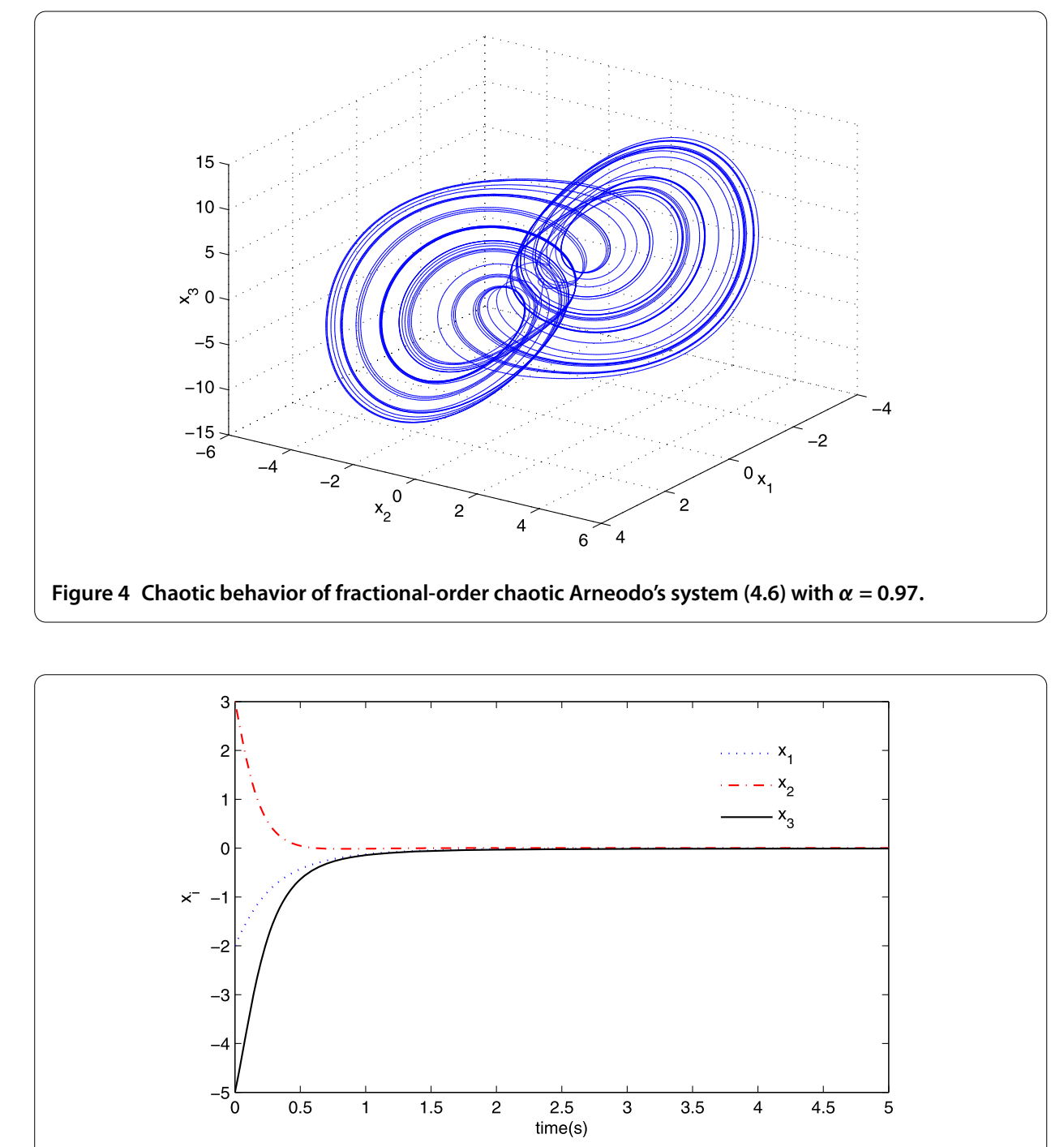

Figure 5 Asymptotic stabilization of system (4.6) with adaptive feedback gains.

feedback gains $k_{i}$ are chosen as $x(0)=(-2,3,-5)$ and $\left(k_{1}(0), k_{2}(0), k_{3}(0)\right)=(1,2,4)$. Thus, all the conditions of Theorem 3.3 are satisfied, and we can conclude that the controlled system is asymptotically stable. The simulation results shown in Figures 5 and 6 validate the theoretical analysis.

\section{Conclusions}

In this paper, we propose some stabilization criteria for a large class of fractional-order nonautonomous systems, for which quadratic Lyapunov functions are always valid. The proposed stability criteria can be seen as an extension and improvement of the existing results in the literatures [16-20,32]. It has been also shown that our proofs of the theorems are simple and straightforward, and the stability conditions are more convenient for testing. Our future work includes the applications of the fractional Lyapunov direct method and fractional inequality techniques in the stabilization of more general fractional-order nonlinear systems. 


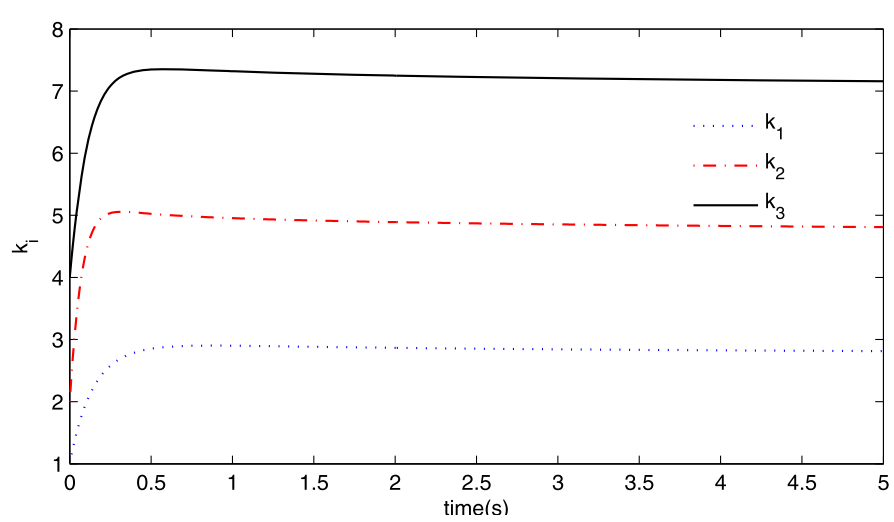

Figure 6 The evolution of the adaptive feedback gains.

\section{Acknowledgements}

This research has been supported by the 'Chunhui Plan' Cooperative Research for Ministry of Education (Grant No. Z2016133), the Scientific Research Foundation of the Education Department of Sichuan Province (Grants Nos. 16ZB0163, 17ZA0364), the Key Scientific Research Fund Project of Xihua University (Grant No. Z17124), the National Natural Science Foundation of China (Grant No. 11402214), the Open Research Fund of Key Laboratory of Automobile Engineering of Sichuan Province (Grant No. szj2016-017), the Open Research Subject of Artificial Intelligence Key Laboratory of Sichuan Province (Grant No. 2017RYJ03), the Open Research Fund of Key Laboratory of Automobile Measurement and Control \& Safety of Sichuan Province (Grant No. szjj2017-074), and the Open Research Fund of Key Laboratory of Numerical Simulation of Sichuan Province (Grant No. 2017KF004).

\section{Competing interests}

The authors declare that they have no competing interests.

\section{Authors' contributions}

All authors contributed equally to the writing of this paper. All authors read and approved the final manuscript.

\section{Author details}

'School of Technology, Xihua University, Chengdu, 610039, China. ${ }^{2}$ Key Laboratory of Fluid and Power Machinery, Ministry of Education, Xihua University, Chengdu, 610039, China. ${ }^{3}$ School of Electrical Engineering, Southwest Jiaotong University, Chengdu, 610031, China. ${ }^{4}$ School of Automobile and Transportation, Xihua University, Chengdu, 610039, China. ${ }^{5}$ School of Electrical Engineering and Electronic Information, Xihua University, Chengdu, 610039, China.

\section{Publisher's Note}

Springer Nature remains neutral with regard to jurisdictional claims in published maps and institutional affiliations.

\section{Received: 4 September 2017 Accepted: 28 December 2017 Published online: 15 January 2018}

\section{References}

1. Valdes-Parada, F, Ochoa-Tapia, J, Alvarez-Ramirez, J: Effective medium equations for fractional Fick's law in porous media. Physica A 373, 339-353 (2007)

2. Singh, J, Kumar, D, Sunil Kumar, R: An efficient computational approach for time-fractional Rosenau-Hyman equation. Neural Comput. Appl. (2017, in press)

3. Magin, R, Ortigueira, MD, Podlubny, P, Trujillo, J: On the fractional signals and systems. Signal Process. 91(3), 350-371 (2011)

4. Singh, J, Kumar, D, Al Qurashi, M, Baleanu, D: A novel numerical approach for a nonlinear fractional dynamical model of interpersonal and romantic relationships. Entropy 19, Article ID 375 (2017)

5. Wu, GC, Baleanu, D: Image encryption technique based on fractional chaotic time series. J. Vib. Control 22, 2092-2099 (2016)

6. Kumar, D, Singh, J, Baleanu, D: A fractional model of convective radial fins with temperature-dependent thermal conductivity. Rom. Rep. Phys. 69(1), Article ID 103 (2017)

7. Yang, XJ, Machado, JAT, Cattani, C, Gao, F: On a fractal LC-electric circuit modeled by local fractional calculus. Commun. Nonlinear Sci. Numer. Simul. 47, 200-206 (2017)

8. Singh, J, Kumar, D, Al Qurashi, M, Baleanu, D: A new fractional model for giving up smoking dynamics. Adv. Differ. Equ. 2017, Article ID 88 (2017)

9. Luo, Y, Chen, YQ: Fractional order [proportional derivative] controller for a class of fractional order systems. Automatica 45(10), 2446-2450 (2009)

10. Xu, Q, Zhuang, SX, Liu, SJ, Xiao, J: Decentralized adaptive coupling synchronization of fractional-order complex-variable dynamical networks. Neurocomputing 186, 119-126 (2016)

11. Li, CP, Zhang, FR: A survey on the stability of fractional differential equations. Eur. Phys. J. Spec. Top. 193(1), 27-47 (2011) 
12. Wu, GC, Baleanu, D, Zeng, SD: Finite-time stability of discrete fractional delay systems: gronwall inequality and stability criterion. Commun. Nonlinear Sci. Numer. Simul. 57, 229-308 (2017)

13. Baleanu, D, Wu, GC, Bai, YR, Chen, FL: Stability analysis of Caputo-like discrete fractional systems. Commun. Nonlinear Sci. Numer. Simul. 48, 520-530 (2017)

14. Aghababa, MP: A Lyapunov-based control scheme for robust stabilization of fractional chaotic systems. Nonlinear Dyn. 78, 2129-2140 (2014)

15. Wu, GC, Baleanu, D, Xie, HP: Chaos synchronization of fractional chaotic maps based on the stability condition. Physica A 460, 374-383 (2016)

16. Wen, XJ, Wu, MZ, Lu, JG: Stability analysis of a class of nonlinear fractional-order systems. IEEE Trans. Circuits Syst. II, Express Briefs 55(11), 1178-1182 (2008)

17. Tavazoei, MS: Comments on "Stability analysis of a class of nonlinear fractional order systems". IEEE Trans. Circuits Syst. II, Express Briefs 56(6), 519-520 (2009)

18. Chen, L, Chai, Y, Wu, R, Yang, J: Stability and stabilization of a class of nonlinear fractional-order systems with Caputo derivative. IEEE Trans. Circuits Syst. II, Express Briefs 59(9), 602-606 (2012)

19. Zhang, R, Tian, G, Yang, S, Cao, H: Stability analysis of a class of fractional order nonlinear systems with order lying in $(0,2)$. ISA Trans. 56, 102-110 (2015)

20. Chen, L, He, Y, Chai, Y, Wu, R: New results on stability and stabilization of a class of nonlinear fractional-order systems. Nonlinear Dyn. 75(4), 633-641 (2014)

21. Li, Y, Chen, YQ, Podlubny, I: Mittag-Leffler stability of fractional order nonlinear dynamic systems. Automatica 45(8), 1965-1969 (2009)

22. Li, Y, Chen, YQ, Podlubny, I: Stability of fractional-order nonlinear dynamic systems: Lyapunov direct method and generalized Mittag-Leffler stability. Comput. Math. Appl. 59(5), 1810-1821 (2010)

23. Baleanu, D, Ranjbar, A, Sadati, S, Delavari, R, Abdeljawad, T, Gejji, V: Lyapunov-Krasovskii stability theorem for fractional systems with delay. Rom. J. Phys. 56(5-6), 636-643 (2011)

24. Wu, GC, Baleanu, D, Luo, WH: Lyapunov functions for Riemann-Liouville-like fractional difference equations. Appl. Math. Comput. 314, 228-236 (2017)

25. Tarasov, VE: No violation of the Leibniz rule. No fractional derivative. Commun. Nonlinear Sci. Numer. Simul. 18(11), 2945-2948 (2013)

26. Benzaouia, A, Hmamed, A, Mesquine, F, Benhayoun, M, Tadeo, F: Stabilization of continuous-time fractional positive systems by using a Lyapunov function. IEEE Trans. Autom. Control 59(8), 2203-2208 (2014)

27. Agarwal, R, Hristova, S, O'Regan, D: Lyapunov functions and strict stability of Caputo fractional differential equations. Adv. Differ. Equ. 2015, 346 (2015)

28. Trigeassou, JC, Maamri, N, Sabatier, J, Oustaloup, A: A Lyapunov approach to the stability of fractional differential equations. Signal Process. 91(3), 437-445 (2011)

29. Hu, JB, Lu, GP, Zhang, SB, Zhao, LD: Lyapunov stability theorem about fractional system without and with delay. Commun. Nonlinear Sci. Numer. Simul. 20(3), 905-913 (2015)

30. Aguila-Camacho, N, Duarte-Mermoud, MA, Gallegos, J: Lyapunov functions for fractional order systems. Commun. Nonlinear Sci. Numer. Simul. 19(9), 2951-2957 (2014)

31. Duarte-Mermoud, MA, Aguila-Camacho, N, Gallegos, J, Castro-Linares, R: Using general quadratic Lyapunov functions to prove Lyapunov uniform stability for fractional order systems. Commun. Nonlinear Sci. Numer. Simul. 22(1-3), 650-659 (2015)

32. Liu, S, Jiang, W, Li, X, Zhou, XF: Lyapunov stability analysis of fractional nonlinear systems. Appl. Math. Lett. 51, 13-19 (2016)

33. Podlubny, I: Fractional Differential Equations. Academic Press, San Diego (1999)

34. Agarwal, RP, Belmekki, M, Benchohra, M: A survey on semilinear differential equations and inclusions involving Riemann-Liouville fractional derivative. Adv. Differ. Equ. 2009, Article ID 981728 (2009)

35. DeLellis, P, Bernardo, M, Russo, G: On QUAD, Lipschitz, and contracting vector fields for consensus and synchronization of networks. IEEE Trans. Circuits Syst. I, Regul. Pap. 58(3), 576-583 (2011)

36. Diethelm, K, Ford, NJ, Freed, AD: A predictor-corrector approach for the numerical solution of fractional differential equations. Nonlinear Dyn. 29, 3-22 (2002)

37. Petráš, I: Fractional Order Nonlinear Systems Modeling, Analysis and Simulation. Springer, Berlin (2011)

\section{Submit your manuscript to a SpringerOpen ${ }^{\circ}$ journal and benefit from:}

- Convenient online submission

- Rigorous peer review

- Open access: articles freely available online

- High visibility within the field

- Retaining the copyright to your article

Submit your next manuscript at $>$ springeropen.com 